\title{
基于机器学习算法的夹层玻璃冲击破坏预测模型研究
}

\author{
孟娦然 ${ }^{1,2,3}$, 王星尔 ${ }^{1,2,3,4}$, 杨 健 ${ }^{1,2,3}$, 徐 涵 ${ }^{1,2,3}$, 岳 峰 ${ }^{1,2}$
}

(1. 上海交通大学 船舶海洋与建筑工程学院, 上海 200240; 2. 上海交通大学 海洋工程国家重点实验室, 上海 200240; 3. 上海交通大学 船舶海洋与建筑工程学院, 上海市公共建筑和基础设施数字化运维重点实验室, 上海 200240; 4. 宁波大学 冲击与安全工程教育部重点实验室, 宁波 315211)

摘 要: 在诸如风致飞射物撞击等刚体冲击作用下，建筑夹层玻璃因自身脆性特征极易破坏。针对这个问题提出了 在刚体冲击下夹层玻璃破坏状态的预测方法，综合考虑了玻璃构型、中间胶层、支撑条件及尺寸等多种设计参数。 首先针对多类夹层玻璃进行往复刚体冲击试验, 建立 567 组 PVB 及 210 组 SGP 的两种不同中间胶层的夹层玻璃试 验数据库; 随后基于鲸鱼优化下的核极限学习机(WOA-KELM)机器学习算法, 建立夹层玻璃破坏状态的预测模型, 并与支持向量机(Support Vector Machine, SVM)及最小二乘支持向量机(Least Squares Support Vector Machine, LSSVM) 建立的相应预测模型进行对比分析。结果表明, WOA-KELM 模型破坏状态预测精度达 $88.45 \%$, 能较好地预测夹层 玻璃的破坏, 满足工程应用的需求, 且预测模型精度及实时性均优于其他模型。

关 键 词: 夹层玻璃; 冲击破坏; 机器学习; 核极限学习机

中图分类号: TQ171 文献标识码: A

\section{Research on Machine Learning Based Model for Predicting the Impact Status of Laminated Glass}

\author{
MENG Yanran ${ }^{1,2,3}$, WANG Xinger ${ }^{1,2,3,4}$, YANG Jian ${ }^{1,2,3}$, XU Han ${ }^{1,2,3}$, YUE Feng ${ }^{1,2}$
}

(1. School of Naval Architecture, Ocean and Civil Engineering, Shanghai Jiao Tong University, Shanghai 200240, China; 2. State Key Laboratory of Ocean Engineering, Shanghai Jiao Tong University, Shanghai 200240, China; 3. Shanghai Key Laboratory for Digital Maintenance of Buildings and Infrastructure, School of Naval Architecture, Ocean and Civil Engineering, Shanghai Jiao Tong University, Shanghai 200240, China; 4. Key Laboratory of Impact and Safety Engineering, Ministry of Education, Ningbo University, Ningbo 315211, China)

\begin{abstract}
Architectural laminated glass exhibits significant vulnerability under hard body impacts such as windborne debris impacts. In this work, a prediction model is proposed for assessing the impact status of laminated glass under hard body impact. Multiple design variables including the glass make-ups, interlayer types, support conditions and size are considered. The impact tests with consecutive impact attempts are first conducted. A comprehensive database encompassing the failure condition of each glass layer is then established. This database has 567 groups of PVB laminated glass data and 210 groups of SGP laminated glass data. A combined WOA-KELM machining learning based model is subsequently developed to predict the impact status of laminated glass. The modelling results are compared with that from SVM and LSSVM based models. The results show that the proposed model
\end{abstract}

收稿日期：2020-04-09; 收到修改稿日期：2020-05-22; 网络出版日期：2020-06-15

基金项目: 国家重点研发计划 (2017YFC0806100); 国家自然科学基金(51908352); 冲击与安全工程教育重点实验室(宁波 大学)开放课题(CJ201906)

National Key Research and Development Program of China (2017YFC0806100); National Natural Science Foundation of China (51908352); Project of Key Laboratory of Impact and Safety Engineering (Ningbo University), Ministry of Education (CJ201906)

作者简介：孟嫣然(1996-), 女, 硕士研究生. E-mail: yrmeng@sjtu.edu.cn MENG Yanran(1996-), female, Master candidate. E-mail: yrmeng@sjtu.edu.cn

通信作者: 杨 健, 教授. E-mail: j.yang.1@sjtu.edu.cn YANG Jian, professor. E-mail: j.yang.1@sjtu.edu.cn 
has a prediction accuracy of $88.45 \%$ in failure status of each glass layer. Such model can well predict the impact status of laminated glass and shows better performance in both accuracy and computation cost than other models.

Key words: laminated glass; impact failure; machine learning; kernel extreme learning machine

夹层玻璃由于添加中间胶层, 与单层玻璃相比 更具安全性, 近年来在建筑幕墙及结构构件领域得 到广泛应用。夹层玻璃的耐冲击设计常采用“牺牲 层”的设计理念 ${ }^{[1-3]}$, 即牺牲外层玻璃抵抗冲击作用, 保留内层玻璃承受外部风荷载, 需要配置好内外层 玻璃的种类和厚度。此外, 中间胶层可对冲击作用 形成缓冲, 并通过粘结作用影响冲击波传递路径, 防止开裂; 在开裂后粘附碎片能继续发挥抵抗冲击 的作用。然而受自身脆性限制, 夹层玻璃在动载作 用下仍具有高易损性。夹层玻璃受刚体冲击后的破 坏是一种难以预测的复杂动态现象, 近年来针对玻 璃在动载作用下的破坏模态开展了较多的试验与数 值研究 ${ }^{[4-10]}$ 。影响夹层玻璃在动载作用下的破坏因 素与其破坏状态存在复杂的非线性关系 ${ }^{[11]}$, 难以通 过简单直观的数学模型构建破坏预测模型。传统的 预测方法如非线性有限元分析, 随着数据量的增加, 预测过程耗时且效率低下。综合而言, 针对夹层玻 璃在动载作用下的破坏状态, 目前尚无可以综合考 虑多种因素的定量预测方法。

机器学习理论的发展为求解高度非线性关系等 复杂问题提供了有效的解决方案 ${ }^{[12-13]}$, 广泛应用于 材料科学和结构工程等领域 ${ }^{[14-18]}$ 。传统神经网络常 采用缓慢梯度下降的方式进行训练学习, 不可避免 地存在训练时间过长、易陷入局部最优值等缺陷。 针对以上算法的弊端, 黄广斌等 ${ }^{[19]}$ 提出了一种单隐 藏层前馈核极限学习机 (Kernel Extreme Learning Machine, KELM), 仅需设置隐藏层节点数, 利用最 小二乘法计算权值, 采用核映射, 取代传统算法的 反复调整更新, 学习速度及泛化能力优势显著。

本研究首先以最小平均碎裂速度测试法 (MMBV)为基础, 考虑多设计参数的影响, 进行大 量落重冲击试验形成基础数据库。为解决 KELM 核 函数参数设置敏感的问题, 通过鲸鱼优化算法(The Whale Optimization Algorithm, WOA) ${ }^{[20]}$ 寻优求得适 用于 KELM 模型的参数。随后, 在分析和量化试验 数据的基础上提出一种基于鲸鱼优化核极限学习机 (WOA-KELM) 的预测模型, 针对刚体冲击作用下夹 层玻璃的破坏状态进行预测分析。最后从计算效率、 预测精度等方面将其与 SVM 及 LSSVM 模型的预测 结果进行比较。本研究所提出的预测模型为玻璃幕 墙的实时监测提供了新的思路, 针对极端天气下夹
层玻璃易受到风致飞射物的冲击产生破坏的隐患进 行预警，在考虑大量数据的前提下可保证预警的及 时性及准确性, 同时为夹层玻璃抗冲击性能设计及 结构优化提供参考。

\section{1 冲击试验数据库}

\section{1 刚体冲击试验设置}

为建立刚体冲击作用下夹层玻璃的破坏状态数 据库, 基于 Kaiser 等 $^{[1]}$ 提出的最小平均碎裂速度测 试法(MMBV)设计刚体冲击试验, 试验设置如图 1 所示。试验采用端部半径为 $40 \mathrm{~mm}$ 的钢球撞击头及 可保持稳定下坠的圆钢柱组成的冲击刚体, 质量为 $13.5 \mathrm{~kg}$, 冲击测试采用逐步增加刚体的冲击速度, 直到试件的内外层玻璃均破碎, 并测定和分析每层 玻璃受冲击时对应的冲击能量及破坏状态。为精确 测定多层玻璃同时碎裂工况下的玻璃开裂顺序, 同 时采用高速相机捕捉开裂过程。

落重冲击试验采用的试件配置情况详见表 1 。 试件设计参数包括: 玻璃种类(钢化玻璃 FTG、半钢 化玻璃 HSG、浮法玻璃 ANG)、中间胶层类型(PVB 及 SGP)及每层厚度、玻璃面板平面尺寸、边界支撑 条件(边缘夹固式及驳接点支式)。对 17 组共计 66 块夹层玻璃进行往复落重冲击试验, 测定每一次冲 击直至破碎的破坏状态, 最终形成 567 组 PVB 和 210 组 SGP 夹层玻璃的有效试验数据集。

\section{2 特征参数相关性分析及数据库建立}

玻璃强度除了与其特定材质相关，同时取决于 加工质量和玻璃表面微观损伤情况。本研究中玻璃 的平均断裂强度参考 VEER 等 ${ }^{[21]}$ 通过四点弯曲试验 统计出的钢化玻璃、半钢化玻璃及浮法玻璃三种类 型玻璃的平均破坏应力, 将其作为各玻璃类型的特 征强度参数, 数据库中材料特征参数如表 2 所示。

为探究输入参数与破坏状态之间的相关性, 本 研究利用受试者操作特性(Receiver Operating Characteristic, ROC)曲线进行综合评估, ROC 曲线是将 系统灵敏度及特异性结合起来综合评价系统诊断准 确度的一种有效的方法 ${ }^{[22]}$, 近年来已有不少研究将 其应用在分类模型评价中 ${ }^{[23-27]}$ 。模型灵敏度以检出 率 TPR(True positive rate)反映, 表示所有实测值为 正的样本中, 被正确判断为正值的比率, 即所有实 

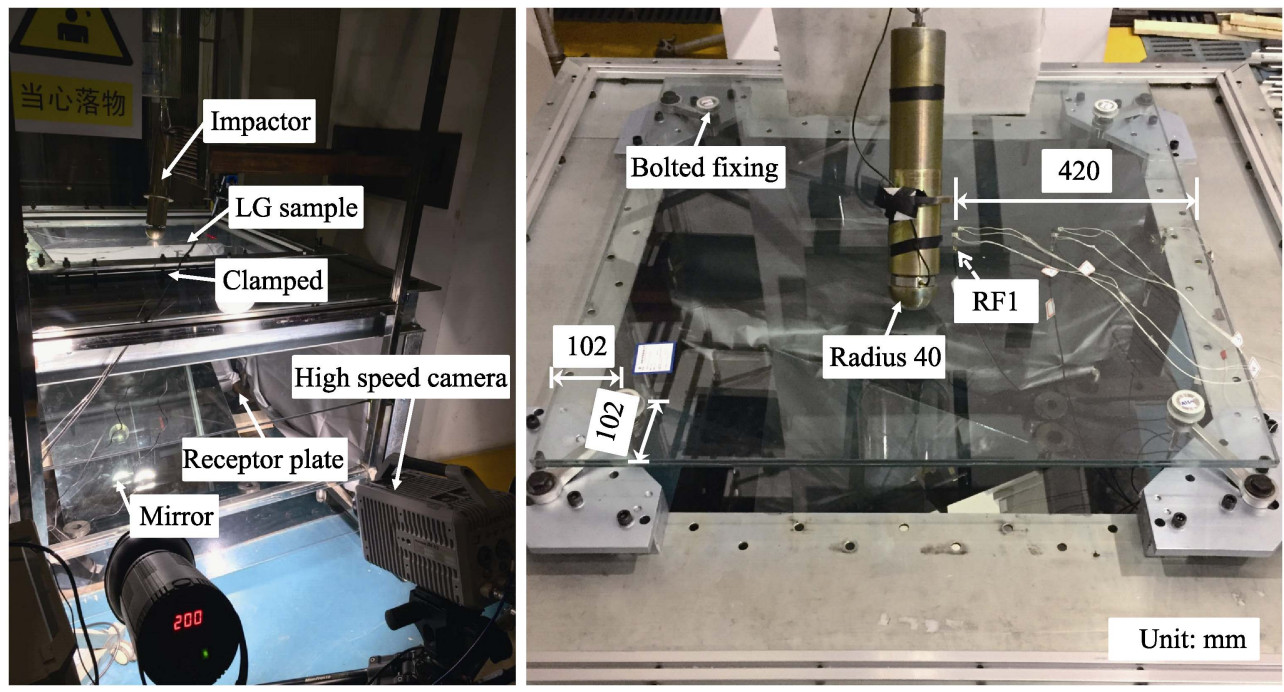

图 1 冲击试验装置图

Fig. 1 Diagram of impact tests equipment

表 1 夹层玻璃试件配置情况

Table 1 Configuration of laminated glass specimens

\begin{tabular}{cccccc}
\hline ID & Material & Make-up (o/m/i) & Dimensional of glass/mm & Support condition & Quantity \\
\hline P01 & FTG/PVB/FTG & $8 / 1.52 / 8$ & $1000 \times 1000$ & Edge clamped & 12 \\
P02 & FTG/PVB/FTG & $8 / 1.52 / 8$ & $1000 \times 1000$ & Bolted connection & Edge clamped \\
P03 & FTG/PVB/FTG & $8 / 1.52 / 8$ & $1500 \times 1500$ & Bolted connection & 3 \\
P04 & FTG/PVB/FTG & $8 / 1.52 / 8$ & $1500 \times 1500$ & Bolted connection & 3 \\
P05 & HSG/PVB/HSG & $8 / 1.52 / 8$ & $1000 \times 1000$ & Bolted connection & 3 \\
P06 & FTG/PVB/FTG & $8 / 0.76 / 8$ & $1000 \times 1000$ & Bolted connection & 3 \\
P07 & FTG/PVB/FTG & $8 / 3.04 / 8$ & $1000 \times 1000$ & Bolted connection & 3 \\
P08 & FTG/PVB/FTG & $8 / 1.52 / 10$ & $1000 \times 1000$ & Bolted connection & 3 \\
P09 & FTG/PVB/FTG & $6 / 1.52 / 10$ & $1000 \times 1000$ & Bolted connection & 3 \\
P10 & FTG/PVB/HSG & $8 / 1.52 / 8$ & $1000 \times 1000$ & Bolted connection & 3 \\
P11 & HSG/PVB/FTG & $8 / 1.52 / 8$ & $1000 \times 1000$ & Edge clamped & 3 \\
S01 & ANG/SGP/FTG & $8 / 3 / 8$ & $1000 \times 1000$ & Edge clamped & 3 \\
S02 & FTG/SGP/ANG & $8 / 3 / 8$ & $1000 \times 1000$ & Bolted connection & 6 \\
S03 & FTG/SGP/FTG & $8 / 3 / 8$ & $1000 \times 1000$ & Bolted connection & 3 \\
S04 & FTG/SGP/FTG & $8 / 3 / 8$ & $1500 \times 1500$ & Bolted connection & 3 \\
S05 & HSG/SGP/FTG & $8 / 3 / 8$ & $1000 \times 1000$ & Bolted connection & 3 \\
S06 & FTG/SGP/FTG & $8 / 5 / 8$ & $1000 \times 1000$ & &
\end{tabular}

表 2 试验数据库材料参数

Table 2 Test database material parameters

\begin{tabular}{ccccccc}
\hline Material parameter & FTG & HSG & ANG & PVB & SGP \\
\hline Density $/\left(\mathrm{kg} \cdot \mathrm{m}^{-3}\right)$ & - & 2500.0 & - & 1100.00 & 950.00 \\
Elasticity modulus/GPa & - & 70.0 & - & 0.15 & 0.30 \\
Poission ratio & - & 0.2 & - & 0.45 & 0.45 \\
Mean failure strength/MPa 157.4 & 104.0 & 42.0 & - & - \\
\hline
\end{tabular}

测破碎玻璃样本中预测出为破碎状态的比率; 特异 性以误检率 FRP(False positive rate)反映, 表示所有 实测值为负的样本中, 预测中被错误判断为正值的
比率，即所有实测完整玻璃样本中预测时错误判断 为破碎状态的比率。以 FPR 为横坐标, TPR 为纵坐 标绘制的 ROC 曲线为一单调曲线, 曲线越凸说明 该模型诊断价值越高。分别将每种输入参数单独做 分类预测, 以 ROC 曲线下面积 AUC 作为量化指标, 其大小反映了此项输入与破坏状态的相关性, 只有 AUC $>0.5$, 此项输入参数对破坏状态的预测准确度 才有贡献。

$$
\begin{gathered}
T P R=T_{\mathrm{P}} /\left(T_{\mathrm{p}}+F_{\mathrm{N}}\right)=T_{\mathrm{P}} /(\text { All Real Positive }) \\
F P R=F_{\mathrm{P}} /\left(F_{\mathrm{p}}+T_{\mathrm{N}}\right)=F_{\mathrm{P}} /(\text { All Real Negative })
\end{gathered}
$$

式中, 预测类别 $T_{\mathrm{P}}$ 为实测值正, 网络预测正; $T_{\mathrm{N}}$ 为 
实测值负, 网络预测负; $F_{\mathrm{p}}$ 为实测值负, 网络预测 正, 为误测; $F_{\mathrm{N}}$ 为实测值正, 网络预测负, 为漏测。

图 2 为单项输入参数的 ROC 曲线, 总体来看, 针对各输入参数均在基准线 $(\mathrm{TPR}=\mathrm{FPR})$ 以上, 面积 AUC 基本大于 0.5 , 反映了所选输入参数提高了分 类的准确度。表 3 中 $A_{n}^{\circ} 、 A_{n}^{\mathrm{i}}$ 分别为外层和内层破坏 状态预测模型的 AUC 值, $n$ 代表输入特征参数编号。 $A_{12}^{0}<0.5$ 是由于外层冲击后的受损状态与内层冲击前 状态之间相关性较低。峰值冲击动能是一个关键变 量, 不在讨论之列。除峰值冲击动能外, 在冲击实验 中玻璃中间胶层类型与外层玻璃破坏状态相关性最 高 $\left(A_{1}^{\circ}=0.605\right)$, 这是由于冲击刚体局部的挤压导致 外层玻璃破裂, 增加中间胶层厚度会提高夹层玻璃 的面外刚度, 可能会更频繁地触发外玻璃层断裂; 玻璃中间胶层类型及厚度与内层玻璃破坏状态相关 性较高, 这是由于冲击作用下底面的周向拉应力波 导致内层玻璃断裂, 而中间胶层会针对这种应力波 起缓冲作用, 其中 $A_{4}^{\mathrm{i}}=0.576, A_{1}^{\mathrm{i}}=0.571$ 。结合试验中 不同开裂顺序下支撑条件对玻璃碎裂刚度的影响, 内层先破坏的情况下采用边缘夹固式支撑条件可使 夹层玻璃碎裂刚度提升 $70 \%$, 结合 $A_{8}^{\mathrm{i}}=0.573$ 可知, 支撑条件与内层玻璃破坏状态相关性最高。

组合 11 种输入参数的预测模型 ROC 曲线如图 3 所示, $A_{13}^{\circ}$ 及 $A_{13}^{\mathrm{i}}$ 均显著高于单项输入参数的 AUC。
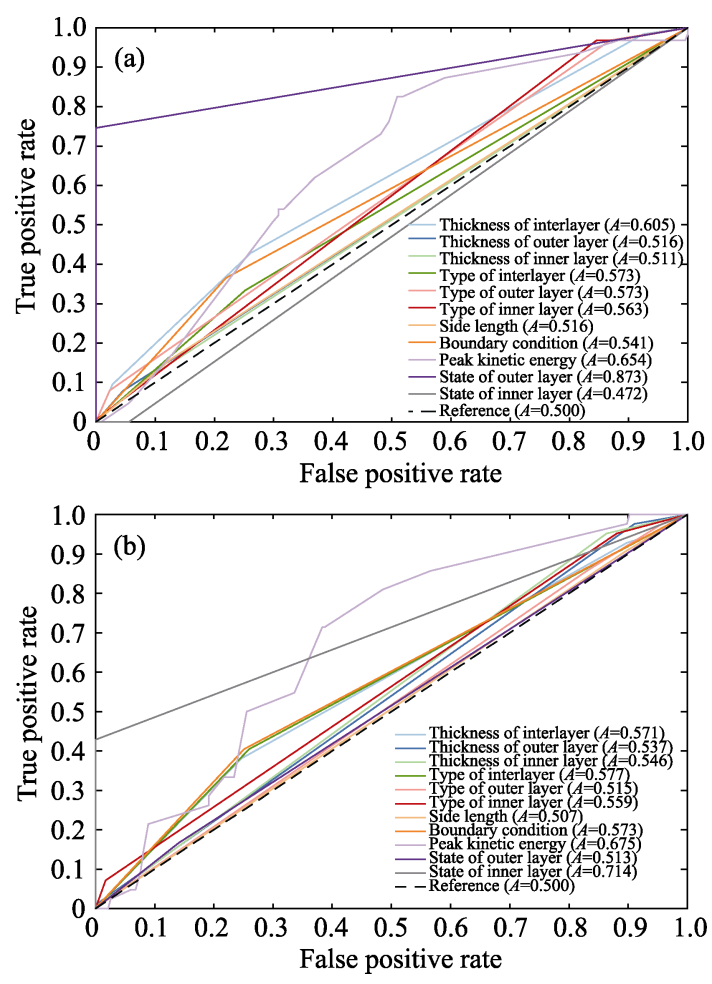

图 2 单项输入参数的 ROC 曲线

Fig. 2 ROC curves of single input parameter (a) Outer layer; (b) Inner layer
表 3 破坏状态预测模型 AUC 值

Table 3 AUC value of the failure status prediction model

\begin{tabular}{cccc}
\hline$n$ & Input parameter & $\begin{array}{c}\text { Outer layer } \\
\text { state AUC }\left(A_{n}^{\circ}\right)\end{array}$ & $\begin{array}{c}\text { Inner layer } \\
\text { state AUC }\left(A_{n}^{\mathrm{i}}\right)\end{array}$ \\
\hline 1 & Thickness of interlayer & 0.605 & 0.571 \\
2 & Thickness of outer layer & 0.516 & 0.537 \\
3 & Thickness of inner layer & 0.511 & 0.546 \\
4 & Type of interlayer & 0.573 & 0.576 \\
5 & Type of outer layer & 0.573 & 0.515 \\
6 & Type of inner layer & 0.563 & 0.559 \\
7 & Side length & 0.516 & 0.507 \\
8 & Boundary condition & 0.541 & 0.573 \\
9 & Peak kinetic energy & 0.654 & 0.675 \\
10 & State of outer layer & 0.873 & 0.513 \\
11 & State of inner layer & 0.472 & 0.714 \\
12 & Multiple input & 0.916 & 0.842 \\
\hline
\end{tabular}
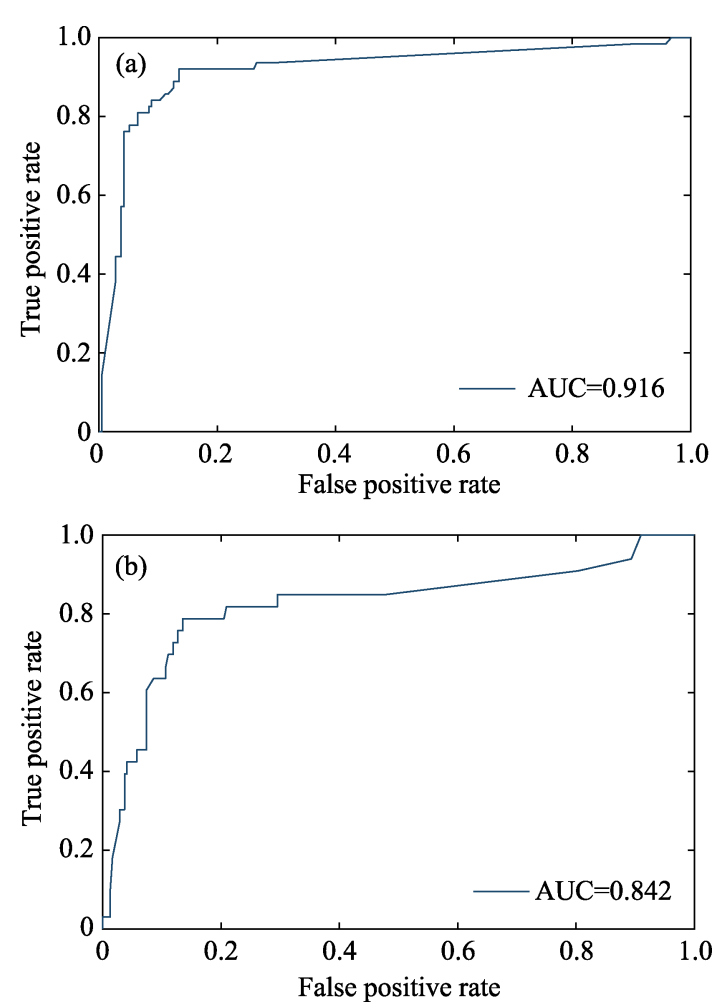

图 3 联合输入参数的 ROC 曲线

Fig. 3 ROC curves of integrated input parameters (a) Outer layer; (b) Inner layer

外层破坏状态预测模型 ROC 曲线如图 3(a)所示, $A_{13}^{\circ}=0.916$, 预测模型准确度较高; 考虑到误测比漏 测对夹层玻璃安全预警的影响更大, 内层预测模型 (图 3(b))ROC 曲线 TPR 远高于 FPR, 即检出率高于 误检率, 此结果较为保守。

综上所述, 夹层玻璃在刚体冲击下的破坏状态 相关性最高的输入参数为支撑条件及中间胶层材料 特性, 通过比对试验结果初步验证了预测模型的有 效性, 且 11 种输入参数的组合预测效果较好。 


\section{2 核极限学习机理论与鲸鱼优化算法}

由上述分析结果得到输入特征参数库, 并验证 了其与输出的相关性, 表明预测模型的有效性。据 此建立基于核极限学习机的预测模型, 并利用鲸鱼 优化算法对其核参数进行优化，对受刚体冲击下夹 层玻璃的破坏状态进行预测。

\section{1 核极限学习机模型}

核极限学习机(KELM)是一种多输入多输出的 单隐层前馈神经网络 ${ }^{[28]}$, 可以随机生成网络中的隐 藏层偏差和输入权重, 并使用 Moore-Penrose 广义逆 确定输出权重, 在泛化能力及学习速度上有显著优 势。其由三层网络结构构成: 输入层、隐藏层和输出 层, 网络模型如图 4 所示, $x_{i}$ 为输入数据集, $K\left(x, x_{i}\right)$ 为 内核函数, $\beta_{j}$ 为连接隐藏节点和输出节点的输出权重。

\section{2 鲸鱼优化算法}

核参数的优化对于 KELM 的性能也起着关键性 作用, 近年来多采用群智能优化算法等方法进行优 化 ${ }^{[29-30]}$ 。鲸鱼优化算法(WOA) 是由 Mirjalili 等 ${ }^{[20]}$ 提 出的一种新型元启发式算法, 该算法具有调整参数 少、操作简单和易于跳出局部最优的优点。图 5 为 优化过程中定义初始位置 $\boldsymbol{P}_{\boldsymbol{i}}(X, Y),(i=1,2, \cdots N)$ 的搜 索代理在搜索空间中求得最优解 $\boldsymbol{P}^{*}\left(X^{*}, Y^{*}\right)$ 的寻优过 程, $A$ 为系数向量。

\section{3 建立 WOA-KELM 预测模型}

在综合考虑的前提下, 采用内外玻璃种类及厚 度、中间胶层类型和厚度、边界条件、玻璃面板尺 寸、冲击动能及玻璃冲击前状态等 11 项指标作为该 模型的输入向量, 建立夹层玻璃受刚体冲击作用后 破坏状态预测模型。夹层玻璃破坏后的状态与其材 料参数及冲击条件可以简化为式(3)所示的函数关 系表达式。

$$
\begin{aligned}
& {\left[X_{\mathrm{o}}^{\prime}, X_{\mathrm{i}}^{\prime}\right]^{T}=} \\
& \quad f_{\mathrm{E}}\left(T_{\mathrm{o}}, T_{\mathrm{m}}, T_{\mathrm{i}}, S_{\mathrm{o}}, S_{\mathrm{i}}, E_{\mathrm{m}}, L_{\mathrm{G}}, B_{\mathrm{C}}, E_{\mathrm{p}}, X_{\mathrm{o}}, X_{\mathrm{i}}\right)
\end{aligned}
$$

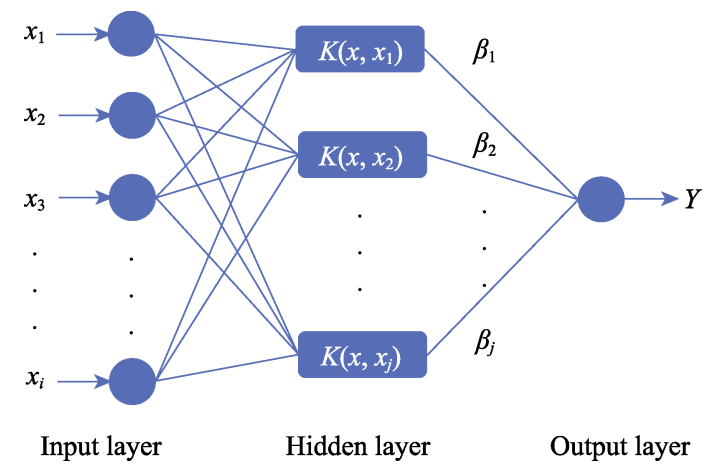

图 4 KELM 网络结构

Fig. 4 The network structure of KELM

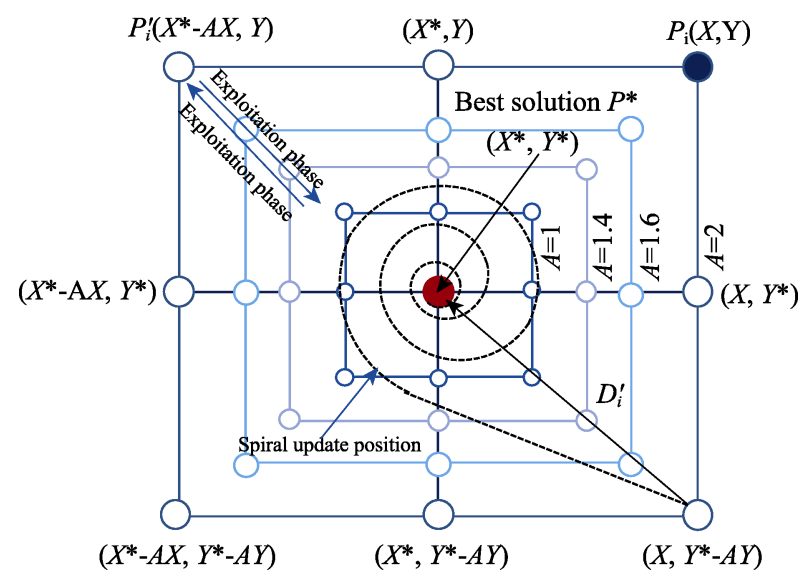

图 5 WOA 寻优过程

Fig. 5 WOA optimization process

式中左侧项， $X_{0}^{\prime}, X^{\prime}$ 分别为外、内层玻璃受冲击后 的破坏状态。式中右侧项, $T_{\mathrm{o}}, T_{\mathrm{m}}, T_{\mathrm{i}}$ 分别为外层、中 间胶层和内层厚度; $S_{0}, S_{\mathrm{i}}$ 分别为外、内层的平均破 坏强度; $E_{\mathrm{m}}$ 为中间胶层的弹性模量; $L_{\mathrm{G}}$ 为面板边长; $B_{\mathrm{C}}$ 为玻璃面板边界支撑条件; $E_{\mathrm{p}}$ 为冲击峰值动能; $X_{0}, X_{\mathrm{i}}$ 分别为外、内层玻璃受冲击前的状态。

夹层玻璃破坏状态的输入及输出信号由内外层 玻璃状态共同构成，玻璃整体状态按照受损程度归 为四类并进行量化处理, 即: 完整无损 (I 级)、外层 破坏(II 级)、内层破坏(III 级)及全部破坏(IV 级)。

夹层玻璃受刚体冲击作用下的破坏状态及特征 响应预测模型计算流程如图 6 所示, 具体步骤如下:

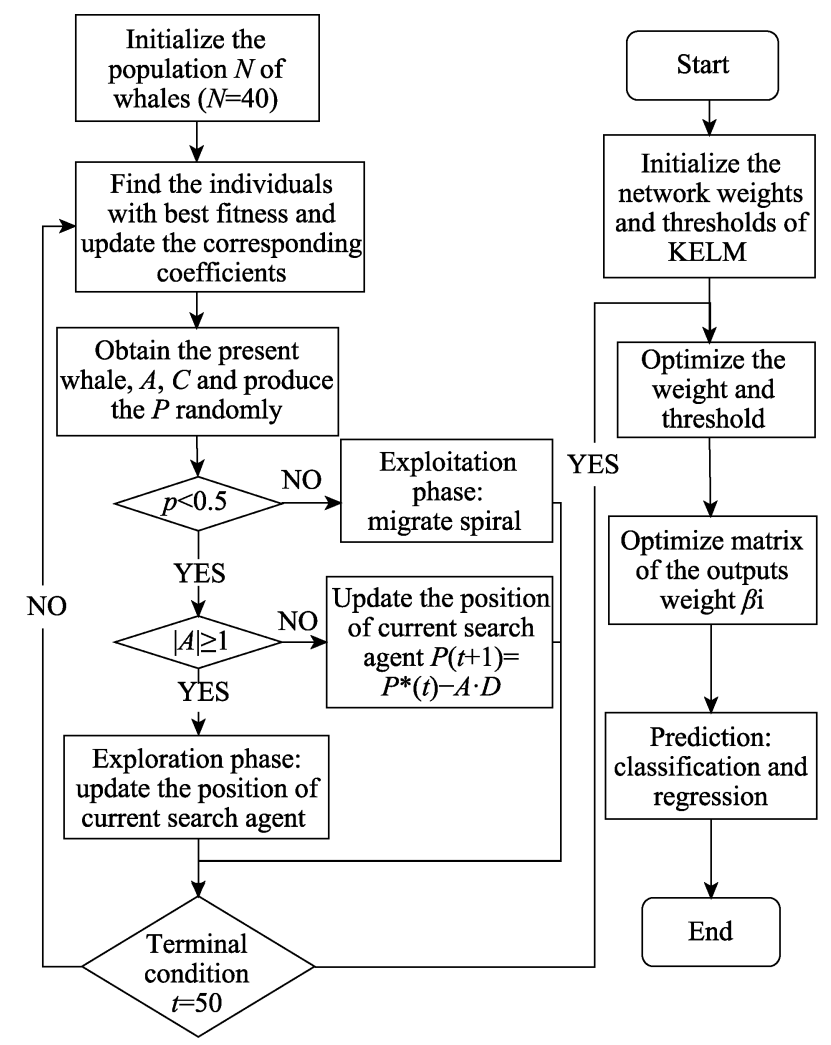

图 6 WOA-KELM 流程图

Fig. 6 WOA-KELM flow chart 
步骤 1)由于各玻璃试件的冲击数据存在一定的 差异性, 同时为减少各输入参数数量级不同对网络 训练的影响, 针对特征数据进行预处理, 用标准化 处理消除数据库各维数据间数量级差别;

步骤 2)高斯核学习性能极强, 具有较好的非线 性映射能力, 在分类问题上鲁棒性好, 选取高斯核 函数作为 KELM 的核函数, 针对预测模型 $y=f_{\mathrm{E}}(x)$ 及 试验数据库中的训练试件 $\left(x_{i}, y_{i}, i=1,2 \ldots N\right)$ 进行训练 学习, 获得输入向量映射 $\hat{y}=f_{\text {kelm }}(x)$ 及初始输出权 重 $\beta_{\text {int, }}$, 选择均方差作为适应度的目标函数 fitness $=(1 / N) \sum_{i=1}^{N}\left(y_{i}-\hat{y}_{i}\right)^{2}$;

步骤 3)利用 WOA 算法对 KELM 模型中的核参 数 $\gamma$ 及罚系数 $C$ 进行寻优, 最小化适应度函数, 迭 代后得到最优输出权重 $\beta^{\prime}$;

步骤 4)根据 KELM 最优输出权重 $\beta^{\prime}$, 计算测试 数据的预测输出。

\section{3 结果分析}

以冲击试验数据库为研究对象, 随机提取 500 组试验试件数据作为训练集对预测模型进行训练, 剩余 277 组数据作为测试集。KELM 采用高斯核, 将 测试数据输入模型, 针对夹层玻璃受冲击后的破坏 状态进行预测, 仿真环境如表 4 所示。

为了与 KELM 模型的预测性能进行对比, 本研 究选取 SVM、LSSVM 模型作为对比模型。三者均 具有较好的泛化性能和学习速度, 具有相同含义的 参数, 但针对特定问题时因不同的自身限制会产生 不同的运算结果, 进行三者的比对可为建立模型和 选择预测方法提供更多的可能性, 为工程应用提供 最优解。

针对夹层玻璃受刚体冲击作用下破坏状态进行 预测, WOA 参数优化如图 7 所示, 罚系数 $C$ 及核参 数 $\gamma$ 越大则容错率越小, 预测精度越高, 但易过拟 合; 越小则容错率越大, 泛化能力提高, 但易欠拟 合。适应度函数 fitness $=53.95$ 时参数取最优值, 罚 系数 $C=8041, \gamma=0.1131$, 预测结果如表 5 所示。

表 4 仿真环境

Table 4 Simulation environment

\begin{tabular}{cc}
\hline Item & Detailed settings \\
\hline Hardware & \\
CPU & Quad-core intel core i7-4850HQ \\
Frequency & $2.3 \mathrm{GHz}$ \\
RAM & $16 \mathrm{~GB} 1600 \mathrm{MHz}$ DDR3 \\
Hard drive & $500 \mathrm{~GB}$ \\
Operating system & MacOS \\
\hline
\end{tabular}

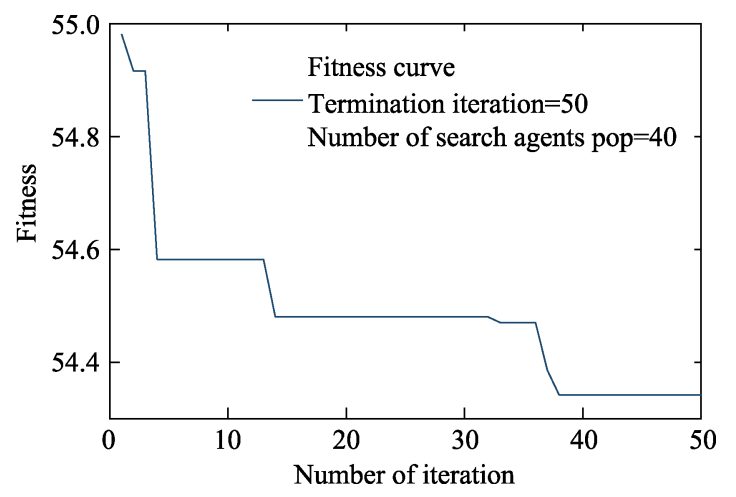

图 7 WOA 优化过程

Fig. 7 WOA optimization process

表 5 玻璃破坏状态预测结果

Table 5 Prediction results of glass failure status

\begin{tabular}{cccc}
\hline Model & $\begin{array}{c}\text { Computing } \\
\text { time/ms }\end{array}$ & $\begin{array}{c}\text { Training } \\
\text { accuracy/\% }\end{array}$ & $\begin{array}{c}\text { Testing } \\
\text { accuracy/\% }\end{array}$ \\
\hline WOA-KELM & 10.62 & 93.80 & 88.45 \\
SVM & 367.87 & 92.80 & 87.00 \\
LSSVM & 65.28 & 89.20 & 85.56 \\
\hline
\end{tabular}

基于 WOA-KELM 的玻璃破坏状态预测结果 (图 8)表明, 输出的预测值均良好地逼近了实测数 据, 预测模型训练精度达到 $93.80 \%$, 测试精度达到 $88.45 \%$ 。为实现夹层玻璃的安全预警, 预测破坏的 关键在于判断内层玻璃的状态。图 8 中箭头所示位 置表示实测值误判下对应的预测值, 其中部分 III 级 (内层破坏) 实测值对应的预测值被分类至 IV 级(全 部破坏), 即将结果归类于最危险的状态, 此结果较 为保守; 部分 IV 级实测数据对应的预测数据被分 类至 II 级(外层破坏), 在实际应用中, 当外层玻璃 受损后为避免内层继续破损，应立即采取安全措施， 避免漏测。结果表明: 277 个测试样本中准确预测 245 次, 漏测 13 次, 19 次误判中保守估测 8 次, 说明 本研究提出的基于 WOA-KELM 预测模型能较好地 预测夹层玻璃在刚体冲击下的破坏状态, 且出现误 测情况时能获得较为保守的预测数据, 满足实际应 用的要求。

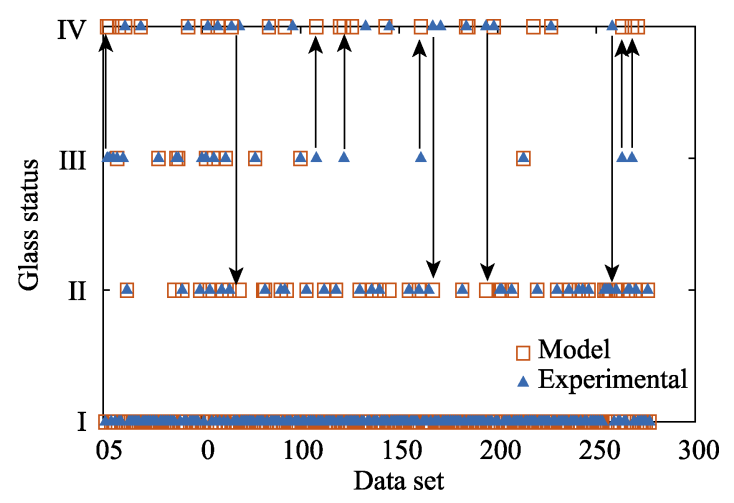

图 8 WOA-KELM 玻璃破坏状态预测结果

Fig. 8 WOA-KELM glass failure status prediction results 


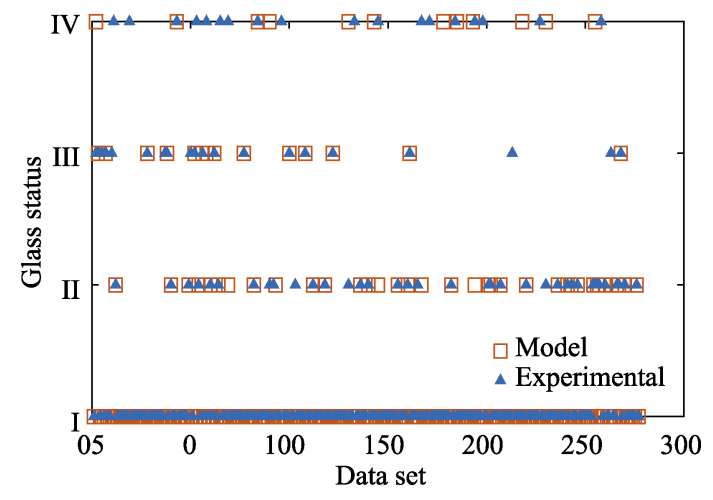

图 9 SVM 模型玻璃破坏状态预测结果

Fig. 9 SVM glass failure status prediction results

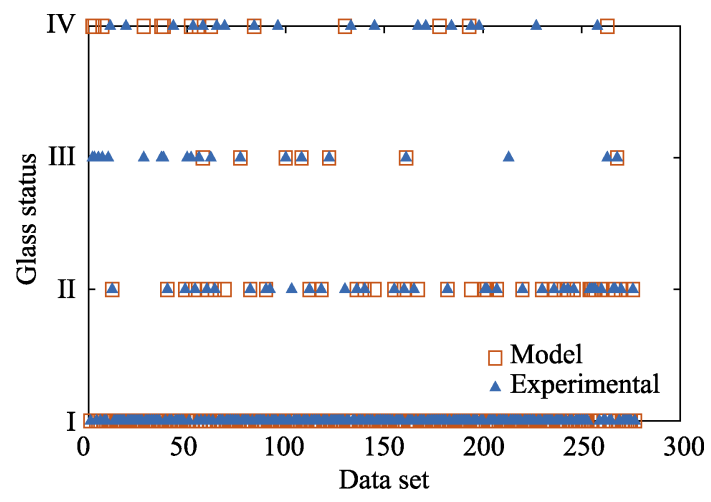

图 10 LSSVM 模型玻璃破坏状态预测结果

Fig. 10 LSSVM glass failure status prediction results

图 9 为 SVM 准确预测 241 次, 漏测 25 次, 11 次误判中保守估测 5 次; 图 10 为 LSSVM 准确预测 237 次, 漏测 22 次, 18 次误判中保守估测 14 次。在 表 5 对比结果中, SVM 测试精度达到 87.0\%, 略低于 WOA-KELM，计算时间是 KELM 的 35 倍; LSSVM 测试精度达到 $85.6 \%$, 为三者最低, 计算时间是 KELM 的 6 倍。KELM 和 SVM 模型总体思路相似, 但前者大大缩短了计算时间, LSSVM 提高了部分求 解速度, 可以用于数据较多的冲击试验库, 但易因 未获得全局最优解而降低精度。综上, 针对于夹层 玻璃破坏状态的分类预测中, WOA-KELM 具有较 高的精度，且在计算时间上优势显著。

\section{4 结论}

1)通过受试者操作特性(ROC) 曲线对夹层玻璃 冲击作用下的 11 个输入特征参数的相关性进行了分 析, 结合试验现象与参数分析表明, 中间胶层厚度、 类型及外层玻璃类型是外层破坏状态的主要影响变 量, 中间胶层类型、厚度及边界支撑条件是内层玻璃 破坏状态的主要影响变量; 边界支撑条件及中间胶 层对夹层玻璃抗冲击性能影响较大，同时证明所提 出的预测模型有效地结合了多输入变量的有效性;
2)基于冲击试验数据库建立基于鲸鱼优化算法 核极限学习机(WOA-KELM)预测模型, 升求得的破 坏状态精度达 $88.45 \%$ ，该方法得到的预测模型精度 满足实际应用需求;

3)WOA-KELM 模型的预测精度及计算时间均 优于 SVM 及 LSSVM 模型, 针对该数据库的平均计 算时间为 $10 \mathrm{~ms}$, 满足夹层玻璃极端天气风致飞射物 冲击下安全预警的实时性要求。

\section{参考文献:}

[1] KAISER NATHAN D, BEHR RICHARD A, MINOR JOSEPH E, et al. Impact resistance of laminated glass using "sacrificial ply" design concept. Journal of Architectural Engineering, 2000, 6(1): 24-34.

[2] SAXE TIMOTHY J, BEHR RICHARD A, MINOR JOSEPH E, et al. Effects of missile size and glass type on impact resistance of "sacrificial ply” laminated glass. Journal of Architectural Engineering, 2002, 8(1): 24-39.

[3] WANG XING-ER, YANG JIAN, LIU QIANG, et al. Experimental investigations into SGP laminated glass under low velocity impact. International Journal of Impact Engineering, 2018, 122: 91-108.

[4] ZHANG XI-HONG, HAO HONG, MA GUO-WEI. Laboratory test and numerical simulation of laminated glass window vulnerability to debris impact. International Journal of Impact Engineering, 2013, 55: 49-62.

[5] CHEN SHUN-HUA, ZANG MENG-YAN, WANG DI, et al. Finite element modelling of impact damage in polyvinyl butyral laminated glass. Composite Structures, 2016, 138: 1-11.

[6] WANG XING-ER, YANG JIAN, LIU QING-FENG, et al. A comparative study of numerical modelling techniques for the fracture of brittle materials with specific reference to glass. Engineering Structures, 2017, 152: 493-505.

[7] MOHAGHEGHIAN IMAN, WANG Y, JIANG L, et al. Quasi-static bending and low velocity impact performance of monolithic and laminated glass windows employing chemically strengthened glass. European Journal of Mechanics-A/Solids, 2017, 63: 165-186.

[8] ALTER CHRISTIAN, KOLLING STEFAN, SCHNEIDER JENS. An enhanced non-local failure criterion for laminated glass under low velocity impact. International Journal of Impact Engineering, 2017, 109: 342-353.

[9] ZHANG YANG-MEI, WANG XING-ER, YANG JIAN. Experimental study of multiple layered SGP laminated glass under hard body impact. Journal of Inorganic Materials, 2018, 33(10): 1110-1118.

[10] WANG XING-ER, YANG JIAN, WANG FEILIANG, et al. Simulating the impact damage of laminated glass considering mixed mode delamination using FEM/DEM. Composite Structures, 2018, 202: 1239-1252.

[11] LIU XIAO-GEN, BAO YI-WANG, SONG YI-LE, et al. The calculation method and influence factor to the natural frequency of laminated glass. Bulletin of the Chinese Ceramic Society, 2008, (27)5: 918-923.

[12] DAS SANTANU, SRIVASTAVA ASHOK N, CHATTOPADHYAY ADITI. Classification of Damage Signatures in Composite Plates using One-class SVMs. 2007 IEEE Aerospace Conference. MT, USA. 2007: 1-19.

[13] LI HONG-NAN, GAO DONG-WEI, YI TING-HUA. Advances in structural health monitoring systems in civil engineering. Advances 
in Mechanics, 2008(02): 151-166.

[14] JIANG ZHEN-YU, ZHANG ZHONG, FRIEDRICH KLAUS. Prediction on wear properties of polymer composites with artificial neural networks. Composites Science and Technology, 2007, 67(2): 168-176.

[15] CHATTERJEE SANKHADEEP, SARKAR SARBARTHA, HORE SIRSHENDU, et al. Particle swarm optimization trained neural network for structural failure prediction of multistoried RC buildings. Neural Computing and Applications, 2017, 28(8): 2005-2016.

[16] XU HAN, YANG JIAN, WANG XING-ER, et al. Application of back propagation neural network on debonding prediction of glass curtain walls with concealed frames. Journal of the Chinese Ceramic Society, 2019, 47(8): 1073-1079.

[17] SHENG MING-JIAN, CHEN PU-HUI, QIAN YI-BIN. An estimating method of compressive strength of composite laminates after low-velocity impact. Journa of Shanghai Jiao Tong University, 2019, 53(10): 1182-1186.

[18] ZHANG YONG-ZHEN, TONG XIAO-YAN, YAO LEI-JIANG, et al. Acoustic emission pattern recognition on tensile damage process of $\mathrm{C} / \mathrm{SiC}$ composites using an improved genetic algorithm. Journal of Inorganic Materials, 2020, 35(5): 593-600.

[19] HUANG GUANG-BIN, DING XIAO-JIAN, ZHOU HONG-MING. Optimization method based extreme learning machine for classification. Neurocomputing, 2010, 74(1/2/3): 155-163.

[20] MIRJALILI SEYEDALI, LEWIS ANDREW. The whale optimization algorithm. Advances in Engineering Software, 2016, 95: 51-67.

[21] VEER FA, LOUTER PIETER CHRISTIAAN, BOS FP. The strength of annealed, heat-strengthened and fully tempered float glass. Fatigue \& Fracture of Engineering Materials \& Structures, 2009, 32(1): $18-25$.
[22] FAWCETT TOM. An introduction to ROC analysis. Pattern Recognition Letters, 2006, 27(8): 861-874.

[23] HUANG J, LU J, LING C X. Comparing Naive Bayes, Decision trees, and SVM with AUC and Accuracy. Third IEEE International Conference on Data Mining. Melbourne, FL, USA, USA. 2003: 553-556.

[24] BARAKAT N, BRADLEY A P. Rule Extraction from Support Vector Machines: Measuring the Explanation Capability Using the Area under the ROC Curve. 18th International Conference on Pattern Recognition (ICPR'06). 2006: 812-815.

[25] ZHANG X, JIANG C. Improved SVM for Learning Multi-Class Domains with ROC Evaluation. 2007 International Conference on Machine Learning and Cybernetics. Hong Kong, China. 2007: 2891-2896.

[26] TESFAMARIAM SOLOMON, LIU ZHENG. Earthquake induced damage classification for reinforced concrete buildings. Structural Safety, 2010, 32(2): 154-164.

[27] GUI GUO-QING, PAN HONG, LIN ZHI-BIN, et al. Data-driven support vector machine with optimization techniques for structural health monitoring and damage detection. KSCE Journal of Civil Engineering, 2017, 21(2): 523-534.

[28] HUANG GUANG-BIN, WANG DIAN HUI, LAN YUAN. Extreme learning machines: a survey. International Journal of Machine Learning and Cybernetics, 2011, 2(2): 107-122.

[29] HO SL, YANG SHI-YOU, NI GUANG-ZHENG, et al. A particle swarm optimization-based method for multiobjective design optimizations. IEEE Transactions on Magnetics, 2005, 41(5): 1756-1759.

[30] YANG XI-YUN, GUAN WEN-YUAN, LIU YU-QI, et al. Prediction intervals forecasts of wind power based on PSO-KELM. Proceedings of the CSEE, 2015, 35(S1): 146-153. 